\title{
Carcinoma Epidermoide Primario de la Mama: Una Infrecuente Entidad Clínico-Patológica
}

\author{
Primary Squamous Cell Carcinoma of the Breast: A Rare Clinicopathological Entity
}

*,** Oscar Tapia E.

TAPIA, E. O. Carcinoma epidermoide primario de la mama: una infrecuente entidad clínico-patológica. Int. J. Morphol., 29(3):825$829,2011$.

RESUMEN: El carcinoma epidermoide (CE) puro primario de la mama es una rara entidad clínico-patológica, que representa menos del 0,1\% de todos los carcinomas de mama, conformada en su totalidad por células escamosas malignas que no presentan relación anatómica con la piel adyacente de la mama. Su etiopatogenia, pronóstico y tratamiento son motivo de controversias. Presentamos 1 caso de CE puros primario de la mama diagnosticado en la Unidad de Anatomía Patológica del Hospital Hernán Henríquez Aravena de la ciudad de Temuco, Chile.

PALABRAS CLAVE: Carcinoma de células escamosas; Mama; Cáncer de mama.

\section{INTRODUCCIÓN}

El carcinoma epidermoide (CE) puro primario de la mama es una rara entidad clínico-patológica, que representa menos del $0,1 \%$ de todos los carcinomas de mama (Toikkanen, 1981; Gupta et al., 2006; Behranwala et al., 2003; Flikweert et al., 2008).

La denominación CE puro primario denota que la neoplasia esta compuesta en su totalidad por células escamosas malignas que no presentan relación anatómica con la piel adyacente de la mama y se ha descartado el probable origen secundario del tumor (cuello uterino, esófago, etc) (Macia et al., 1989; Siegelmann-Danieli et al., 2005).

La etiopatogenia, pronóstico y tratamiento de esta neoplasia son actualmente motivo de controversias Flikweert et al.; Murialdo et al., 2009; Moisidis et al., 2002; Rostock et al., 1984; Shigekawa et al., 2007; Bhatt \& Fernando, 2009).

Presentamos un caso de CE puro primario de la mama diagnosticado en la Unidad de Anatomía Patológica del Hospital Hernán Henríquez Aravena de la ciudad de Temuco, Chile.

\section{RELATO DEL CASO}

Mujer de 77 años, menarquia a los catorce años, paridad de doce hijos vivos, a los veinte años primer embarazo, tiempo total de lactancia aproximado de ciento cuarenta meses y menopausia a los cincuenta y dos años. Consultó por nódulo autopesquizado en mama izquierda, constatandose al examen clínico en el cuadrante superior externo lesión nodular, indurada y adherida de aproximadamente 4 x 5,5 cm. La mamografía bilateral demostró en la mama izquierda masa radiopaca de 4,5 x 4,3 cm de contornos irregulares y con calcificaciones intratumorales (Figs. 1A-B). En la ecotomografía se aprecia lesión quística de contornos irregulares y pared en partes engrosada, de contenido anecogénico (Fig. 1C). Se completa estudio con biopsia de aguja gruesa (BAG) informando el estudio anatomopatológico tejido glandular mamario infiltrado por proliferación neoplásica maligna compuesta por células poligonales, con núcleos pleomórficos e hipercromáticos, formación de puentes intercelulares y presencia de focos de queratinización con perlas córneas compatible con carcinoma epidermoide queratinizante infiltrante de la mama.

Se efectuó mastectomía total con disección ganglionar axilar nivel I y II demostrando el estudio histopatológico una lesión quística irregular de 5,5 x $4 \mathrm{~cm}$, de

\footnotetext{
* Departamento de Anatomía Patológica. Facultad de Medicina. Universidad de La Frontera, Temuco, Chile.

** BIOREN (Scientific and Technological Bioresource Nucleus), Universidad de La Frontera, Temuco, Chile.
} 


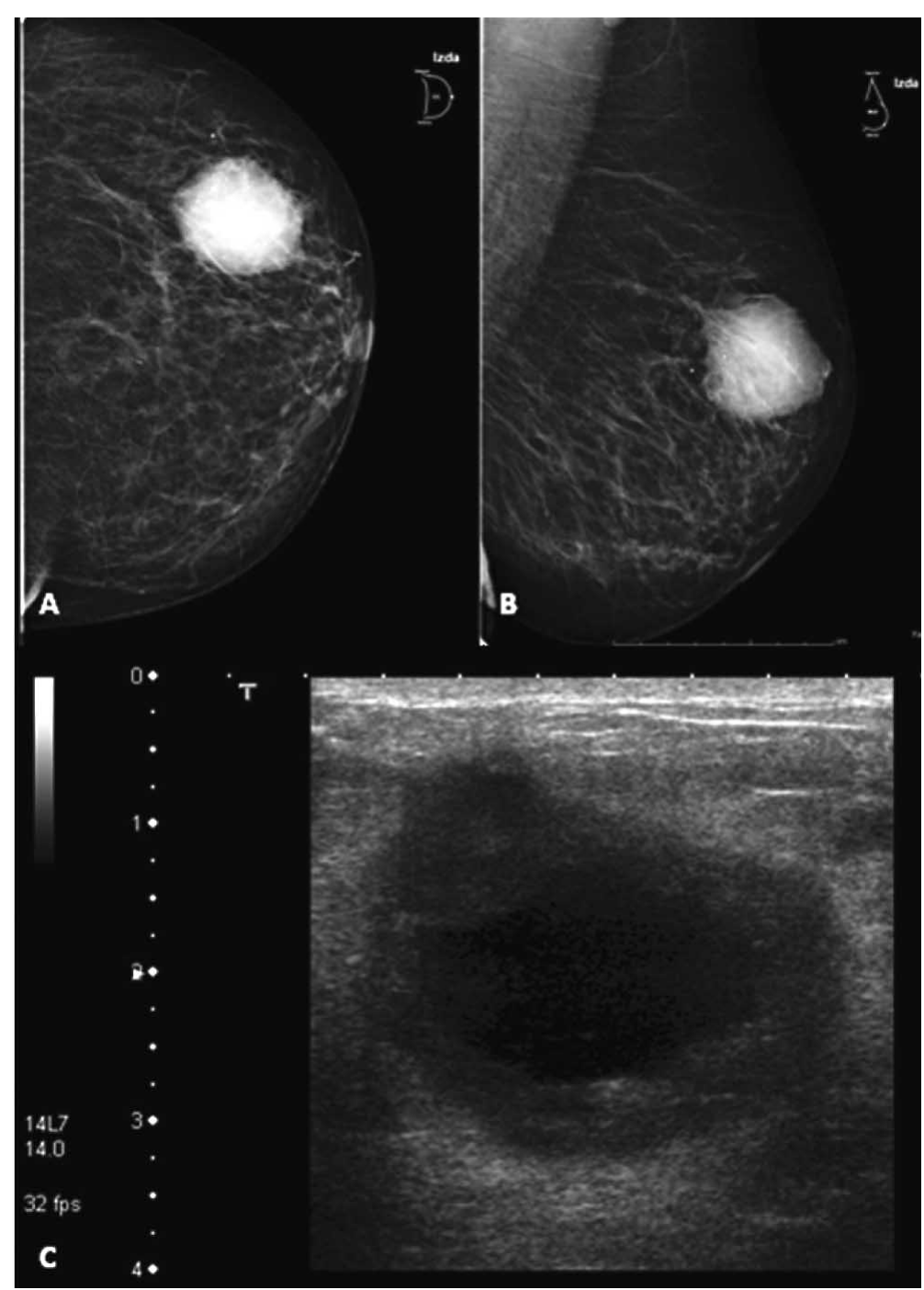

Fig. 1. A. Vesícula biliar con mucosa irregular y nodular con áreas pardo amarillentas en partes acartonadas y zonas violáceas de aspecto hemorrágico. B. Superficie de corte, evidencia pared engrosada de hasta $1,5 \mathrm{~cm}$ con áreas blanquecinas irregulares e induradas.
Fig. 2. Tejido glandular mamario con lesión quística unilocular de paredes irregulares en partes engrosada y ubicada a $1 \mathrm{~cm}$. de borde quirúrgico antero-superior. superficie interna trabeculada y parcialmente rellena por material líquido amarillento (Fig. 2). Al examen microscópico, se reconoce pared de lesión quística con proliferación neoplásica compuesta en su totalidad por células poligonales escamosas, con núcleos pleomórficos, hipercromáticos y vesiculosos, abundantes mitosis atípicas, formación de puentes intercelulares y presencia de focos de queratinización con perlas córneas compatible con carcinoma epidermoide puro de la mama, grado III de Elston. No se observó compromiso tumoral de piel, pezón ni bordes quirúrgicos de sección (Fig. 3). En el tejido adiposo axilar se encontraron 20 ganglios linfáticos, todos ellos sin evidencias de neoplasia. El estudio inmunohistoquímico para receptores hormonales (estrógenos y progesterona) y Her2-neu resultaron negativos. Con el estudio de diseminación se concluye un cáncer de mama T3N0M0. Dado el tamaño de la lesión y grado histológico se indicó quimioterapia (6 FAC) y radioterapia a la pared torácica, encontrándose actualmente completando la quimioterapia.

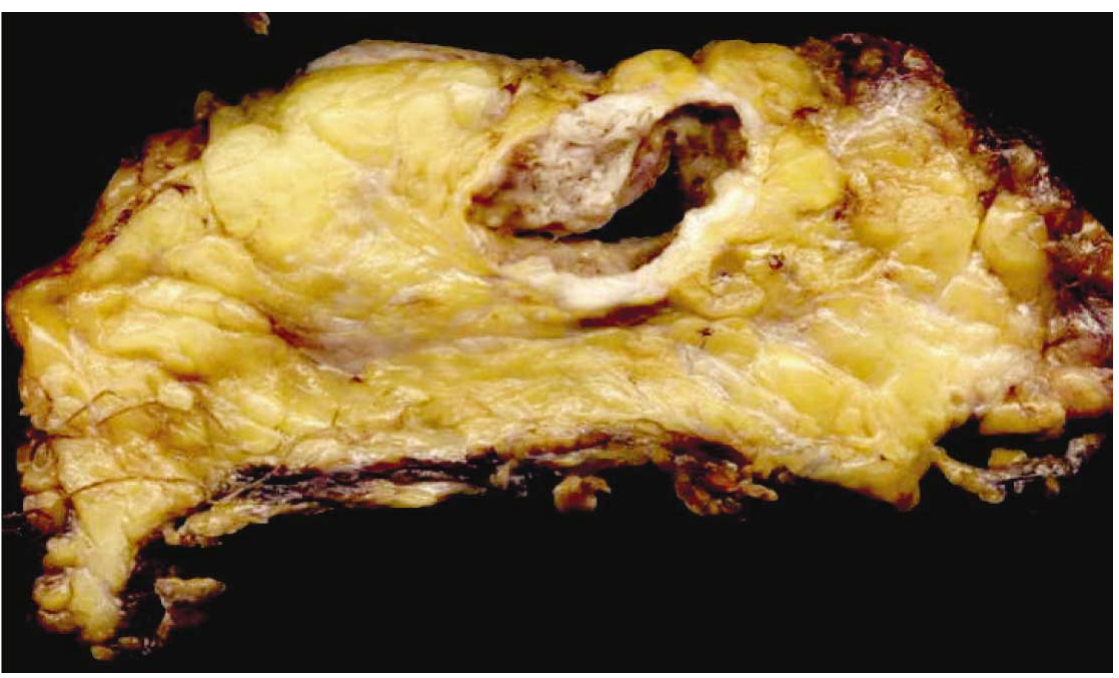



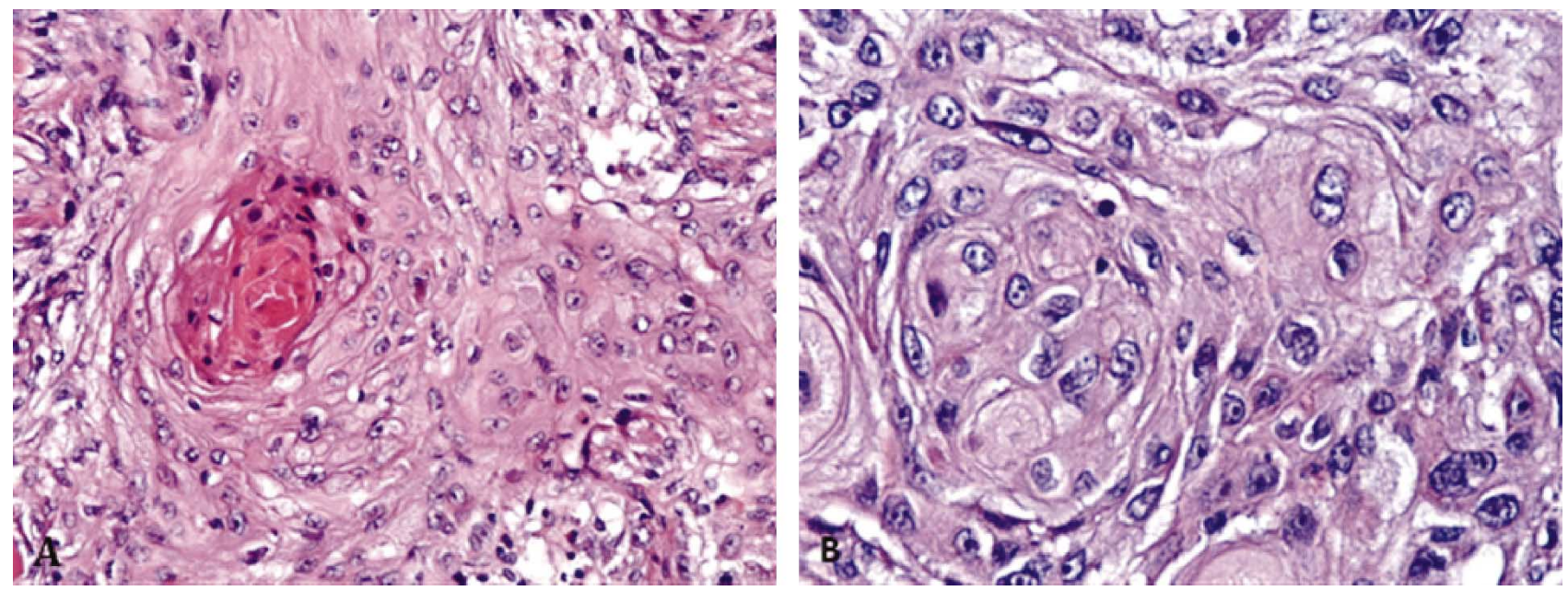

Fig.3. A (HE, 200x) proliferación neoplásica compuesta por nidos infiltrativos de células escamosas con formación de perlas córneas. B (HE, 400x) células tumorales con hipercromasia nuclear, pleomorfismo, cromatina vesiculosa y nucleólo prominente.

\section{DISCUSIÓN}

De acuerdo a la clasificación de la Organización Mundial de la Salud (OMS) el CE de la mama corresponde a un tipo de carcinoma metaplásico, representando la forma pura del CE menos del 0,1\% de todos los carcinomas de mama (Toikkanen; Gupta et al.; Behranwala et al.; Flikweert et al.; Tavassoli \& Devilee, 2003).

Para su diagnóstico deben cumplirse los siguientes criterios clínico-morfológicos: ausencia de otro componente neoplásico maligno que no sea de tipo escamoso, ausencia de relación anatómica del tumor con la piel adyacente de la mama y exclusión clínica de la existencia de otro tumor primario que pudiese haber metastizado a la mama (cuello uterino, esófago, pulmón, laringe, entre otros) (Siegelmann-Danieli et al.; Macia et al.).

La patogénesis del CE primario de la mama es compleja, pues este epitelio no se encuentra normalmente en el tejido mamario; planteándose que esta neoplasia surge luego de un cambio metaplásico en un carcinoma ductal convencional, hecho que explica la existencia de formas mixtas que presentan carcinoma ductal convencional con proporciones variables de áreas escamosas, representando por tanto el CE puro un carcinoma ductal con metaplasia escamosa completa (Behranwala et al.; Murialdo et al.; Stevenson et al., 1996).

Estos tumores afectan generalmente a mujeres de edad avanzada, son habitualmente grandes $(>4 \mathrm{~cm})$ y en la mitad de los casos se presentan como lesiones quísticas (Gupta $e t$ al.; Murialdo et al.; Shigekawa et al.; Hasleton et al., 1978; Cornog et al., 1971), explicado por el rápido crecimiento tumoral con posterior necrosis central, presentando el caso reportado estas características clínico-morfológicas (77 años, tumor T3 con degeneración quística central). Los hallazgos a la mamografía son indistinguibles de un carcinoma ductal, mientras que en la ecotomografía se presenta habitualmente como una masa hipoecogénica con áreas quísticas complejas que en ocasiones puede confundirse con abscesos mamarios (Siegelmann-Danieli et al.; Murialdo et al.; Shigekawa et al.). El estudio histopatológico demuestra una proliferación neoplásica de alto grado compuesta por nidos, confluentes e infiltrativos de células escamosas con o sin diferenciación córnea, donde la mayoría de estos tumores no muestran sobre-expresión de Her2-neu ni positividad de los receptores hormonales (Gupta et al.; Behranwala et al.; SiegelmannDanieli et al.; Murialdo et al.; Cardoso et al.).

El tratamiento de los CE primarios de mama no difiere sustancialmente de la de los otros tipos histológicos de carcinomas mamarios, incluyendo cirugía, quimioterapia, radioterapia y/o terapia hormonal; sin embargo, dado lo infrecuente de esta entidad, no existe un consenso para el tratamiento de esta neoplasia (Flikweert et al.; Murialdo et al.; Shigekawa et al.).

La literatura disponible reporta que entre el 10-30\% de las pacientes con CE de la mama presentan metástasis ganglionares, cuestionando algunos autores la necesidad de efectuar la disección ganglionar; sin embargo, con la finalidad de tener una adecuada etapificación de la enfermedad se aconseja realizarla (Gupta et al.; Flikweert et al.; Murialdo et al.), en este caso se realizó disección axilar sin demostrarse 
metástasis ganglionares. Por otro lado, la terapia adyuvante convencional para carcinomas ductales no ha mostrado diferencias estadísticas significativas en cuanto a recurrencia y supervivencia para $\mathrm{CE}$, mientras que en pacientes que recibieron tratamiento neoadyuvante no se obtuvo respuesta; reportándose, sin embargo, una buena respuesta a cisplatino y 5-fluorouracilo en una paciente con enfermedad metastásica (Murialdo et al.; Moisidis et al.; Cardoso et al.; Aparicio et al., 2008; Hiramatsu et al., 2007). Del mismo modo, el rol de la radioterapia para estos tumores es también controversial, mencionando la literatura la frecuente radiorresistencia de esta neoplasia. La terapia hormonal en tanto, generalmente no está indicada, pues como se mencionó anteriormente estos tumores son habitualmente receptores de estrógenos y progesterona negativos (Gupta et al.; Behranwala et al.; Siegelmann-Danieli et al.; Murialdo et al.; Cardoso et al.).
Tampoco existe acuerdo en cuanto a su pronóstico, y debido a su baja frecuencia es difícil realizar estudios que permitan evaluar este aspecto, sin embargo, se compara su comportamiento biológico al de carcinomas ductales poco diferenciados, reportándose en distintos estudios tasas de supervivencia a 5 años de 39-67\% (Behranwala et al.; Murialdo et al.; Moisidis et al., 2002; Bhatt \& Fernando; Menes et al., 2003; Hennessy et al., 2005).

Finalmente podemos concluir que el CE puro primario de la mama es una infrecuente entidad clínico-patológico que afecta generalmente a mujeres de edad avanzada, siendo su pronóstico pobre. Por otro lado, la baja frecuencia hace necesario estudios colaborativos multicéntricos que permitan evaluar terapias adecuadas para perfiles tumorales específicos.

TAPIA, E. O. Primary squamous cell carcinoma of the breast: a rare clinicopathological entity. Int. J. Morphol., 29(3):825-829, 2011.

SUMMARY: Pure primary squamous cell carcinoma (SCC) of the breast is a rare clinicopathological entity, representing less than $0.1 \%$ of all breast carcinomas, composed entirely of malignant squamous cells do not exhibit anatomical relationship with the skin overlying to the breast. Its etiopathogenesis, prognosis and treatment is controversial. We present a case of pure primary SCC of the breast diagnosed at the Unit of Pathology, Hospital Hernán Henríquez Aravena.

KEY WORDS: Squamous cell carcinoma; Breast; Breast cancer.

\section{REFERENCIAS BIBLIOGRÁFICAS}

Aparicio, I.; Martínez, A.; Hernández, G.; Hardisson, D. \& De Santiago, J. Squamous cell carcinoma of the breast. Eur. J. Obstet. Gynecol. Reprod. Biol., 137(2):222-6, 2008.

Bhatt, L. \& Fernando, I. Primary squamous cell carcinoma of the breast: achieving long-term control with cisplatinbased chemotherapy. Clin. Breast Cancer, 9(3):187-8, 2009.

Behranwala, K. A.; Nasiri, N.; Abdullah, N.; Trott, P. A. \& Gui, G. P. Squamous cell carcinoma of the breast: clinicopathologic implications and outcome. Eur. J. Surg. Oncol., 29(4):386-9, 2003.

Cardoso, F.; Leal, C.; Meira, A.; Azevedo, R.; Mauricio, M. J.; Leal da Silva, J. M.; Lopes, C. \& Pinto Ferreira, E. Squamous cell carcinoma of the breast. Breast, 9(6):3159, 2000.

Cornog, J. L.; Mobini, J.; Steiger, E. \& Enterline, H. T. Squamous carcinoma of the breast. Am. J. Clin. Pathol., 55(4):410-7, 1971.
Flikweert, E. R.; Hofstee, M. \& Liem, M. S. Squamous cell carcinoma of the breast: a case report. World J. Surg. Oncol., 6:135, 2008.

Gupta, C.; Malani, A. K.; Weigand, R. T. \& Rangineni, G. Pure primary squamous cell carcinoma of the breast: a rare presentation and clinicopathologic comparison with usual ductal carcinoma of the breast. Pathol. Res. Pract., 202(6):465-9, 2006.

Hasleton, P. S.; Misch, K. A.; Vasudev, K. S. \& George, D. Squamous carcinoma of the breast. J. Clin. Pathol., 31(2):116-24, 1978.

Hennessy, B. T.; Krishnamurthy, S.; Giordano, S.; Buchholz, T. A.; Kau, S. W.; Duan, Z.; Valero, V. \& Hortobagyi, G. N. Squamous cell carcinoma of the breast. J. Clin. Oncol., 23(31):7827-35, 2005.

Hiramatsu, K.; Kato, K.; Hirata, A.; Matsuba, H.; Hara, T.; Ito, T.; Miyata, T.; Akagawa, T.; Kutsuna, Y.; Machiki, Y. \& Fujioka, S. A resected case of squamous cell carcinoma of the breast successfully treated by FU 
pluscisplatin (CDDP) adjuvant therapy against recurrent metastases. Gan. To. Kagaku Ryoho., 34(3):443-6, 2007.

Macia, M.; Ces, J. A.; Becerra, E. \& Novo, A. Pure squamous carcinoma of the breast. Report of a case diagnosed by aspiration cytology. Acta Cytol., 33(2):201-4, 1989.

Menes, T.; Schachter, J.; Morgenstern, S.; Fenig, E.; Lurie, H. \& Gutman, H. Primary squamous cell carcinoma (SqCC) of the breast. Am. J. Clin. Oncol., 26(6):571-3, 2003.

Moisidis, E.; Ahmed, S.; Carmalt, H. \& Gillett, D. Primary squamous cell carcinoma of the breast. ANZ J. Surg., 72(1):65-7, 2002.

Murialdo, R.; Boy, D.; Musizzano, Y.; Tixi, L.; Murelli, F. \& Ballestrero, A. Squamous cell carcinoma of the breast: a case report. Cases J., 2:7336, 2009.

Rostock, R. A.; Bauer, T. W. \& Eggleston, J. C. Primary squamous carcinoma of the breast: A review. Breast, 10:27-31, 1984.

Shigekawa, T.; Tsuda, H.; Sato, K.; Ueda, S.; Asakawa, H.; Shigenaga, R.; Hiraide, H. \& Mochizuki, H. Squamous cell carcinoma of the breast in the form of an intracystic tumor. Breast Cancer, 14(1):109-12, 2007.

Siegelmann-Danieli, N.; Murphy, T. J.; Meschter, S. C.; Stein, M. E. \& Prichard, J. Primary pure squamous cell carcinoma of the breast. Clin. Breast Cancer, 6(3):2702,2005

Stevenson, J. T.; Graham, D. J.; Khiyami, A. \& Mansour, E. G Squamous cell carcinoma of the breast: a clinical approach. Ann. Surg. Oncol., 3(4):367-74, 1996.

Tavassoli, F. A. \& Devilee, P. World Health Organization Classification of Tumours. Pathology and Genetics of Tumours of the Breast and Female Genital Organs. Lyon, IARC Press, 2003. pp.38-9.

Toikkanen, S. Primary squamous cell carcinoma of the breast. Cancer, 48(7):1629-32, 1981.

\author{
Dirección para correspondencia: \\ Dr. Oscar Tapia E. \\ Departamento de Anatomía Patológica \\ Facultad de Medicina \\ Universidad de La Frontera \\ Manuel Montt 112. \\ Código Postal 478-1176 \\ Temuco \\ CHILE \\ Email: otescalona@gmail.com
}

Recibido : 14-06-2011

Aceptado: 29-07-2011 